\title{
Travail, affects, activité transférentielle
}

Work, affects, transferential activity

\section{Livia Scheller}

\section{OpenEdition}

Journals

Édition électronique

URL : http://journals.openedition.org/activites/853

DOI : 10.4000/activites.853

ISSN : 1765-2723

\section{Éditeur}

ARPACT - Association Recherches et Pratiques sur les ACTivités

\section{Référence électronique}

Livia Scheller, «Travail, affects, activité transférentielle », Activités [En ligne], 10-2 I Octobre 2013, mis en ligne le 15 octobre 2013, consulté le 19 avril 2019. URL : http://journals.openedition.org/ activites/853 ; DOI : 10.4000/activites.853

\section{(2) $\odot \Theta \Theta$}

Activités est mis à disposition selon les termes de la licence Creative Commons Attribution - Pas d'Utilisation Commerciale - Pas de Modification 4.0 International. 


\title{
Travail, affects, activité transférentielle
}

\author{
Livia Scheller \\ CRTD, équipe « Clinique de l'activité », CNAM, Paris, 41, rue Gay Lussac, 75005 Paris \\ livia.scheller@cnam.fr
}

\begin{abstract}
Work, affects, transferential activity. Some interventions in the "clinic of activity" field are motivated by a demand relating to conflicts between managers and employees. This article relates one of these interventions, focusing on affects, emotions and feelings which pass through and mobilise the work activity. This entry constitutes the conceptual object of this paper and the clinical object of the intervention. The article is intended to demonstrate how, starting with the analysis of the job situation, the dialogical activity (which the analysis created) developed another type of activity that we define as "transferential activity". The results of this intervention made it possible to move from the personal affects, proper to the on-going destructive conflicts to the affects understood to stem from the deconstruction of "transpersonal" forms of the job analysis. This transformation made it possible to review the different forms of organization of the service activity in accordance with the employees' suggestions.
\end{abstract}

\section{KEY WORDS}

Co-analysis, dialogical activity, transferential activity, affects, emotions and feelings

\section{1.- Introduction}

Au sein de l'équipe de chercheurs et praticiens de «Clinique de l'activité », ${ }^{1}$ nous sommes engagés dans une tentative de rendre compte de la portée des affects, des émotions et des sentiments (Cosnier, 1994 ; Damasio, 1999 ; Rimé, 2005 ; Vygotski, 1998, 2003) qui traversent, labourent et agitent le domaine du travail. Ce texte propose quelques réflexions sur le thème, à partir de certaines de nos interventions.

D'abord deux constats. Le premier concerne la dimension psychique condensant l'état initial de la demande des professionnels qui s'adressent au clinicien du travail. Il s'agit, le plus souvent, d'une dimension fortement connotée émotionnellement, qui semble avoir trouvé des contours communs à tous les membres du groupe de travail. Nous dirons pour l'instant que la tâche du clinicien consiste à donner à ces « passions collectives » leur genèse. On le fait en co-analysant avec les professionnels concernés les obstacles rencontrés subjectivement et collectivement dans l'exercice de leur activité. Des voies s'ouvrent ainsi à leur dépassement. Ces obstacles manifestent le plus souvent le rapport contradictoire et instable que les individus ressentent entre leur pouvoir d'action dans l'activité à accomplir et le pouvoir de décision sur cette activité, exercé par ceux qui l'organisent officiellement.

Un deuxième constat concerne la manière réaliste par laquelle les professionnels s'approprient des détails opérationnels et relationnels de l'activité qu'ils auront co-analysée. Ces détails deviennent la matière vivante d'où se développent dialogues et controverses entre pairs sur

1 L'équipe de Clinique de l'Activité, dirigée par Y. Clot, opère au sein du CRTD (Centre de Recherches sur le

Travail e le Développement), au CNAM de Paris. 
l'activité commune. Le réalisme des professionnels se manifeste alors dans le choix d'actions transformatrices qui ne sont jamais teintées des couleurs de l'impossible. On dira plutôt qu'elles seront le résultat d'un développement des empêchements vécus dans la conduite de l'activité, une sorte de transformation de l'activité irréalisée en activité possible (Gori, 2002; Vygotski, 2003, 1998), donc réalisable.

\section{2.- L'Analyse de l'activité via l'activité dialogique se transforme en activité transférentielle}

Nos interventions sont basées sur la mise en forme d'un cadre d'analyse de l'activité menée de façon conjointe avec les professionnels qui nous ont directement ou indirectement adressé la demande d'aide. Les interventions se déroulent suivant un cadre censé engager trois activités différentes : une activité d'analyse promouvant une activité dialogique dans un collectif de travail, cette double activité permettant à son tour de faire émerger ce que nous avons appelé une «activité transférentielle». Il s'agit d'une conceptualisation de l'action qui a pris sa source de la problématique du transfert, concept dont l'usage dans notre clinique est justifié par certains phénomènes traversant et définissant nos interventions. Nous en avons approfondi la texture sachant bien que notre cadre est radicalement distingué par son objet et ses méthodes de la clinique psychanalytique, première source de théorisation du concept. C'est alors dans les termes d'«activité transférentielle » qu'il nous a semblé plus pertinent de le définir.

L'activité transférentielle est à envisager comme une d'activité de «transport d'affects » à travers les instances qui structurent toute dimension de travail ayant une histoire, donc la production de formes d'action plus ou moins réglées collectivement. Ces instances, pour le clinicien du travail, constituent la texture de tout métier que, suivant les travaux de Clot (1999, 2009), nous représentons selon quatre dimensions. Nos analyses considèrent en effet que toute activité de travail est toujours portée par un individu : c'est la dimension personnelle. Cet individu, dans son travail, se trouve constamment pris dans une relation à l'autre, qu'il s'agisse des pairs, des supérieurs ou des usagers. Cette relation quotidienne constitue la dimension interpersonnelle. Dimensions personnelle et interpersonnelle sont éventuellement descriptibles. C'est le cas aussi d'une troisième dimension, impersonnelle, concernant la tâche à réaliser. On la définit «impersonnelle » car la tâche prescrite est conçue comme étant à la disposition de n'importe qui la réalisera, elle est en attente d'être personnalisée. Mais il existe une dimension constitutive de toute activité organisée qui, elle, n'est ni observable ni descriptible directement. Il s'agit de l'instance transpersonnelle que l'on peut définir comme issue de l'histoire des savoir-faire déployés au cours du temps dans cette activité, de leur transmission entre les générations qui en ont forgé les formes, les manières de faire et de dire, sans que cela ne soit écrit nulle part.

\section{3.- Le mouvement des affects dans les dimensions personnelles, interpersonnelles, impersonnelles et transpersonnelles d'un métier.}

Comme on tentera de le décrire dans la suite de ce texte, la définition de ces quatre dimensions constitutives de toute activité organisée en métier, et concrètement située, nous sert à montrer comment on peut saisir analytiquement les dynamiques qui déstabilisent la situation de travail qui a fait appel à notre intervention. Nous nous trouvons le plus souvent face à une fragilisation des formes collectives d'action que les professionnels avaient auparavant élaborées pour travailler ensemble efficacement. Ces formes se trouvent maintenant vidées de sens, comme inhabitées. Les individus semblent alors ne plus posséder les moyens d'aborder de façon constructive les désaccords parus dans les dimensions personnelles et collectives de l'activité à réaliser. La situation se nécrose dans des conflits sourds ou bruyants, apparemment insurmontables. 
Dans ces cas, c'est pour nous toujours essentiel de remettre l'activité de travail au centre de la conflictualité ressentie. Nous «forçons » d'une certaine manière nos interlocuteurs à suspendre l'opinion commune qui attribue le plus souvent l'origine des conflits aux traits de caractère difficiles, problématiques, des uns ou des autres (Drida, Engel, \& Litzenberger, 1998 ; Enriquez 1997 ; Pagès, 1993 ; Scheller, 2004) pour replacer l'attention sur leur activité réelle. L'analyse de la situation par l'exercice de discussions entre professionnels sur comment et pourquoi ils ont déployé tel ou tel autre mode d'action est censée rendre plus claires les raisons des dysfonctionnements rencontrés. Se précisent ainsi les dimensions émotionnelles qui secouent négativement la situation. La co-analyse permet alors une déconstruction des significations attribuées aux conflits en cours, grâce à une élaboration de leur sens. Le trajet de cette élaboration suit la dynamique que nous attribuons à l'activité transférentielle: à partir d'une description hautement subjectivée de leur activité personnelle impliquant les opérations et les relations interpersonnelles ponctuant inévitablement leur travail, les professionnels engagés dans le collectif d'analyse se trouvent à créer-trouver d'autres significations aux conflits vécus. Cela est rendu possible grâce aux discussions sur l'activité subjective et collective sollicitées dans le cadre de co-analyse. Ce que l'on vit personnellement comme un obstacle ou ce que l'autre nous fait vivre de façon problématique au travail peuvent être compris comme la manifestation d'un type de conflictualité plus liée à la (dés)organisation du métier, autrement dit à la dimension transpersonnelle qui le caractérise. Les effets de ce «transport » de motions conflictuelles d'une dimension à l'autre se montrent dans les nouvelles liaisons qui apparaissent. Ce qui était affaibli par des conflits dont la signification s'était figée en une version univoque, souvent trop personnalisée, s'ouvre alors. De nouvelles ressources apparaissent, permettant de dénouer ce qui empêchait de travailler de façon sensée et efficace. La redéfinition des tâches, qui définissent la dimension impersonnelle, est aussi l'un des résultats de la transformation visée.

\section{4.- Le destin des affects dans une conceptualisation de l'activité dirigée}

L'activité transférentielle peut révéler comment des conflits vécus personnellement sont le plus souvent le fruit d'un dysfonctionnement de la dimension transpersonnelle. Ce «transport» de signification des conflits ne peut se mettre en mouvement qu'à partir de l'analyse de l'activité subjective dans le cadre du collectif d'analyse. Notre démarche méthodologique est censée mettre en lumière les empêchements propres à l'activité personnelle et collective - et les affects qui les portent. Bien évidemment nos interventions n'ont de sens que si cette conflictualité se manifeste avec une intensité trop importante pour être élaborée spontanément. La démarche méthodologique fait ainsi émerger les obstacles qui barrent l'une des directions dans lesquelles l'activité de travail, subjective et collective, est toujours engagée. Nous parlons de directions de l'activité parce que nous considérons que l'activité de travail est toujours triplement dirigée. Elle est d'abord dirigée vers la tâche à faire, puis vers les autres personnes ayant affaire à cette tâche et, enfin, vers soi-même, puisque toute activité ayant du sens participe, en profondeur, au processus de subjectivation (Clot, 1999, 2001, 2009). En d'autres termes, l'activité de travail engage soi-même à travers la médiation d'un objet à traiter, dans une rencontre inévitable avec l'autre. Or, cette triple direction est nécessairement faite de tensions, elle ne se trouve jamais dans un état d'équilibre. Le mouvement «tensionnel», affectif, entre soi et l'objet auquel on a affaire, et entre soi et les autres qui contribuent à la même activité, participe à la construction d'un «sens de soi » que nous définissons, sans pouvoir en approfondir ici la signification, comme le sens de l'activité propre. Les affects impliqués dans la triple direction de l'activité sont toujours moteur de développement, à condition qu'ils trouvent le moyen de s'articuler de façon créatrice à cette activité. $\mathrm{Si}$, en revanche, ces affects sont ressassés, se fixant dans des émotions conflictuelles, la disponibilité du sujet vis-à-vis du travail collectif est diminuée, le ressentiment, la résignation dominent et l'investissement nécessaire à l'action s'étiole. 
Le cadre théorique et méthodologique de notre approche vise, de fait, à redonner à chaque membre du collectif de travail - et au collectif dans son ensemble - les moyens de saisir, par la dialogie instituée, les rapports que chacun tisse avec l'activité commune, à partir de l'analyse des trois directions de l'activité. La nouvelle compréhension de ces rapports doit permettre de reconsidérer l'origine des conflits destructeurs en cours, pour y trouver, par là même, des réponses actives.

\section{5.- Un service administratif traversé par des passions tristes : contexte et méthode de l'intervention}

Il faut rendre compte plus précisément des processus qu'on vient d'énoncer de façon abstraite et générale. On le fera à partit d'une situation concrète d'intervention, en proposant un exemple particulier d'analyse des mouvements émotionnels qui ont été à la source d'une atmosphère de travail lourde et oppressive au sein d'un service administratif. On verra aussi comment le collectif engagé dans l'analyse de l'activité a su transformer la situation à partir d'une nouvelle perception de la genèse de cette atmosphère.

Nous avons reçu une demande d'intervention concernant un service administratif d'un syndicat qui, à la suite d'un long congé maladie pour cause de dépression d'une salariée, vivait une tension constante entre certains employés et leur direction. Le Comité d'Établissement du syndicat a fait appel à notre équipe pour mieux comprendre les raisons de cette tension et les conflits qu'elle générait. Pour certains membres du CE, la dépression de la salariée semblait indiquer des formes de harcèlement dans le service, le départ précédent de deux autres salariés constituant pour eux un signe précurseur. Mais ce point de vue restait toutefois moins une certitude qu'une interrogation. La secrétaire était tombée malade, disait-on, suite au refus du directeur du service de lui accorder la place de secrétaire de direction qu'elle demandait. Le directeur lui avait dit avec une certaine agressivité, semble-t-il, qu'elle n'en avait pas les compétences. Une autre secrétaire se plaignait du fait qu'elle ne comprenait plus ce qu'elle faisait, car la réorganisation effectuée par la nouvelle direction avait, certes, réduit la charge de travail, trop intense dans le passé, mais en morcelant fortement son activité. De même, la secrétaire qui s'occupait de la maquette du journal interne et du développement informatique avait pris parti, avec ardeur et passion, pour la secrétaire tombée malade, s'identifiant, en quelque sorte, à sa plainte. Comme elle, elle se sentait victime d'une non-reconnaissance malveillante, tendant à la dévalorisation de ses compétences. Les salariés plus jeunes, en revanche, arrivés après la réorganisation du service, faisaient état d'une situation globalement correcte où chacun pouvait faire ce qu'il avait à faire, sans trop de difficultés. La description globale de la situation, non encore soumise à notre cadre méthodologique, était marquée par une charge émotionnelle très passionnelle, qui affectait lourdement les échanges lors de nos premières rencontres avec le groupe de salariés. Des discours farouchement accusateurs circulaient entre les anciens vis-à-vis de leur direction, tandis que les salariés plus jeunes exprimaient, le plus souvent par des attitudes non verbales, l'irritation que cette version des faits provoquait en eux. Bref, la version narrée des conflits en acte était profondément chargée d'un sentiment de haine, d'une part, et d'agacement contre cette haine, d'autre part. Ces «passions tristes »- ressentiment, haine, agacement - semblaient toutes empêcher l'ouverture à une dimension réflexive, nécessaire à la transformation des conflits.

\section{6.- le cadre d'analyse}

Le cadre particulier que nous avons proposé a toutefois permis de retourner cette dimension haineuse en une zone d'élaboration. Nous avons accepté d'intervenir, à la condition que tant les salariés que leurs responsables hiérarchiques s'engagent à redéfinir avec nous, dans des cadres 
d'analyse distingués, l'activité du service et son histoire ${ }^{2}$. Nous avons indiqué les principes de base de notre démarche : chaque participant à l'analyse, s'il accepte volontairement d'y contribuer, doit se rendre disponible pour décrire son activité de travail quotidienne, de façon à rendre intelligibles aux autres, mais aussi à lui-même, les manières d'agir qu'il a mises au point par l'expérience. Bref, tout ce qu'il lui semble «aller de soi », mais qui n'est que le fruit d'une construction subjective plus ou moins longue. Nous avons utilisé dans cette intervention la méthode dite «des instructions au sosie » (Clot, 1995, 1999, 2001 ; Oddone, Re, \& Briante, 1981 ; Scheller, 2001, 2003), dont la consigne, énoncée par l'intervenant, organise l'échange avec chaque participant de la manière suivante : «Demain je vais te remplacer : dis-moi ce que je dois faire pour que personne ne s'aperçoive que j'ai pris ta place». Le professionnel doit répondre aux questions de son remplaçant virtuel, se trouvant ainsi à décrire l'expérience des actes et des gestes qu'il a développée par sa confrontation quotidienne aux tâches prescrites et à tous ceux qui, comme lui, ont affaire à la même activité, ses pairs et ses supérieurs hiérarchiques. À tour de rôle, chaque membre du collectif se prête au même exercice. Le but est de mettre en lumière d'une part les contributions subjectives que chacun apporte à l'activité collective, d'autre part de permettre de retrouver du «commun » dans ce qui apparaît le plus souvent comme des obstacles ou des conflits vécus subjectivement. Dans un deuxième temps, chaque participant à l'analyse doit reprendre le texte des «instructions » qu'il a passées à l'intervenant, enregistrées sur magnétophone et retranscrites, pour réfléchir sur leur contenu. La dernière étape consiste en la relecture subjective et collective de la situation analysée. Cette relecture permet la construction d'une position commune, éventuellement sous la forme d'un document signé par le collectif de travail et adressé au commanditaire de l'intervention.

\section{7.- L'aboutissement de l'analyse}

À tour de rôle, chacun des membres du service technique et administratif de l'organisme a décrit son activité selon les modalités spécifiques que nous avons proposées. Un élément a émergé d'emblée à une première analyse d'ensemble du service : les ancien(ne)s du service étaient les seul(e)s à affirmer que des problèmes étaient bien présents au niveau organisationnel. Leur compréhension n'a été possible qu'en les replaçant dans l'histoire du service, notamment dans les multiples formes dont la division des tâches a été prescrite au cours de la dernière décennie.

L'activité du service concernait, d'une part, l'écriture, la composition et la diffusion du magazine interne à l'organisme social. La description des différentes phases de la fabrication d'un numéro a montré comment des «périodes froides ou chaudes» suivant l'urgence des tâches à accomplir et les délais imposés, étaient vécues différemment selon qu'on pouvait ou pas suivre le déroulement global de la fabrication du journal. Ainsi qu'on le verra plus loin, l'analyse de l'activité de la secrétaire PAO, Mme C., qui faisait partie des «anciennes» du service montrera que si son activité était devenue moins pesante qu'avant, elle s'était en même temps asséchée et vidée de sens. Son activité avait été, en fait, arbitrairement clivée du contexte global de fabrication du journal par une division du travail qui la tenait éloignée des décisions prises sur l'orientation pratique et de contenu du magazine.

Même l'activité plus classiquement administrative avait subi un destin similaire. L'analyse a montré que les tâches des secrétaires étaient, avant la réorganisation, particulièrement nouées aux besoins des responsables hiérarchiques. Après celle-ci, les salariés ont considéré l'organisation des tâches comme étant trop morcelée, sans un rapport clair et partagé à l'activité globale. À ce déséquilibre issu de la nature de l'activité s'ajoutait la persistance d'une

2 Un collectif d'analyse est toujours composé de pairs et ne peut comporter la présence de personnes hiérarchiquement supérieures aux professionnels volontaires. La situation a été ici analysée suivant le point de vue de deux groupes de professionnels : le collectif de secrétaires et le collectif de responsables hiérarchiques. Ces derniers, dont on ne peut pas décrire le travail d'analyse par manque d'espace, ont globalement accepté les résolutions du collectif des salariés, comme on le verra en conclusion. 
dépendance non réglée avec les responsables hiérarchiques. Ainsi qu'on le verra, la réorganisation des tâches n'avait pas remédié à une problématique issue d'une dépendance pathogène entre salariés et dirigeants, propre à l'organisation précédente du service. Cette réorganisation avait même empiré la situation : la rationalisation des tâches avait seulement asséché l'activité des anciennes, les privant d'une source d'intérêt à la vie de l'organisme social qu'elles s'étaient octroyée auparavant, à travers un surplus de travail qu'elles fournissaient à leurs dirigeants. Nous allons illustrer ces points à partir d'un extrait des Instructions au sosie de la secrétaire «PAO » du service, Mme C.

\section{1.- Périodes chaudes et froides, la chaise vide de Bruno, l'exclusion du collectif de rédaction : un exemple de liaison entre détails de l'activité et élaboration de conflits professionnels.}

La maquettiste (PAO) du journal interne, Mme C., avait pris parti pour la secrétaire tombée malade considérant, comme celle-ci, que depuis la réorganisation la direction du service méprisait le travail des salariés. L'extrait que nous présentons rend compte de l'une des difficultés vécues par la secrétaire, Mme $\mathrm{C}$ : c'est moins l'axe de la technicité des tâches qui lui pose problème que l'axe de ses relations de travail, apparemment dense d'obstacles. Cet axe constitue l'une des directions de l'activité que le clinicien essaie d'investiguer à travers la méthode. La maquettiste décrit le démarrage de son activité à l'intervenant, censé la remplacer le lendemain :

Mme C. : Alors, ben! Comment dire ? Alors, la difficulté pour moi, c'est que pour décrire mon travail, c'est que tout dépend de la période que Marthe, je crois, a intitulée : « les pages froides ou les pages chaudes », donc...

Intervenant : ça change radicalement?

Mme C.: Je n'arrive pas à me rendre compte. Parfois, c'est un peu tiède. Et... quand, c'est chaud, c'est que ça se termine, en fait. Alors là, précisément pour dire, Euh ! Si c'était, mettons demain que tu me remplacerais, je ne sais pas ce que tu ferais. Euh ! Dans ce que je viens de faire, éventuellement, je risque... C'est la période froide ou chaude ? Je ne sais pas...

Intervenant : Comment... ? J'ai du mal à comprendre si demain je suis dans des moments de grande charge de travail ou des moments où...?

Mme C : ah ça m'étonne pas que t'as du mal à comprendre parce que j'ai moi-même du mal à comprendre... ben ce qu'il y a c'est que la période froide probablement pour Marthe ${ }^{3}$ et pour moi et les périodes chaudes, je pense que ça se ressemble plus nos périodes chaudes, et comment, mais les périodes froides pour elle et pour moi ne sont pas les mêmes, peut-être qu'elle... la période froide c'est quand elle rentre les textes, quand elle rentre les textes, elle garde les textes, c'est normal ça, elle sait pas dans quel, encore dans quel, ils n'ont pas encore eu les réunions... alors il faut savoir que moi je ne participe à aucune des réunions de préparation du journal!

Intervenant : ... c'est un choix qui me revient?

Mme C : C'est pas un choix qui me revient... qui te revient... absolument pas ! j'ai toujours été demandeuse de participer, dans les tous débuts du magazine, mais alors y a plusieurs années on va dire y a 7 ans j'ai participé même à quelques comités de rédaction où je n'ai rien à dire, mais au moins ça me permettait... ça venait, tout ça vient de la volonté de Bruno ${ }^{4}$ qui de temps en temps avait envie et a envie parfois de m'intégrer et à d'autres moments de ne pas m'intégrer

3 La secrétaire de rédaction (Marthe) avait parlé lors de ses « instructions au sosie » de «pages froides ou chaudes », c'est-à-dire de phases peu ou trop chargées d'activité, pour décrire ses tâches de responsable de rédaction du magazine interne du service.

4 Bruno est le prénom (fictif) du Directeur du journal, responsable hiérarchique de Mme C. 
Intervenant : selon...?

Mme C : selon... il a pas forcément d'arguments, c'est parfois... il me disait dans le temps des choses assez désagréables comme que je n'étais qu'une technique et que j'avais pas... et c'était lui le directeur et puis de toute façon c'était un moment comme ça et à d'autres moments il a été le premier à reconnaître des choses techniques en moi, à d'autres moments à les démolir, donc c'est un peu désarçonnant, déstabilisant, toujours est-il que le comité de rédaction... je n'ai plus participé aux comités de rédaction, j'ai même pas cherché à le demander, il y a au moins deux collectifs de rédaction, j'aurais pu participer aux collectifs de rédaction... Mais depuis la réorganisation on ne m'a plus invitée...

Intervenant : Qui ne « m'a » plus invitée ??

Mme C ...(Pensive) : Oui, Bruno.... Quand il était dans ses bons moments, il venait dans mon bureau, j'aime pas dire mon bureau, ma machine et tout... mais bon, j'ai pas été habituée, faut dire que y a que depuis que je travaille ici que j'ai un bureau, que j'ai une machine mais moi... bon alors ... dans mon bureau, dans le nouveau mobilier, avec le nouveau mobilier, il y avait même, je dois avoir toujours le graphique quelque part, une chaise, la chaise de Bruno... (elle se tait 2 seconds)... ça prouvait que quand même on travaillait ensemble... mais il était question qu'il y ait l'engagement d'une secrétaire de rédaction et Bruno venait et me faisait le compte rendu du collectif, où il me mettait au courant mais ça venait uniquement de sa propre volonté, s'il voulait ou s'il voulait pas, ou s'il estimait que c'était pas nécessaire, ben un jour il pouvait venir et un autre jour ne pas venir mais la preuve est qu'il venait et que c'était vraiment un truc qu'il m'avait... c'est jamais moi qui aurait pu décider, je mets une chaise là quand Bruno vient... non y avait même une rigolade là-dessus... c'était sa chaise, je l'avais même dessinée sur le graphique que j'avais fait pour meubler le bureau et puis... par la suite donc y a l'engagement de Marthe, y a tout ça et je ne vais plus participer ni être invitée à venir parce que là déjà... et puis après je me suis rendue compte que je n'étais plus invitée et ça c'est une chose, une chose! une chose... (expression de rage) que j'ai senti au feeling que c'était pas la peine d'insister que c'était effectivement, il n'y a dans cette équipe que Bruno qui peut refuser de me prendre au collectif, alors ça fait que dans le travail de la réalisation du magazine je suis pratiquement jamais au courant qu'au dernier moment... (elle se tait, visiblement tendue).

Intervenant : Lorsqu'il y a des choses comme ça, c'est-à-dire là alors tu as senti... - c'est pour voir un peu comment je peux faire, moi -... tu as senti quelque part que tu n'étais plus invitée officiellement aux réunions, c'est pour bien saisir comment faire... le fait d'y participer c'est pour moi important, et alors, je me suis pas autorisée à demander ça à Bruno, par exemple ?

La question ouvrira au récit de différents épisodes montrant le caractère interpersonnel que la secrétaire attribuait au déroulement de son activité, presque entièrement adressée à l'avis du responsable du journal interne. La cristallisation de cette adresse «interpersonnelle » dont on voit un exemple dans l'extrait présenté, dominera, chez elle, l'intérêt porté aux compétences techniques développées (au niveau de la fabrication de la maquette). En d'autres termes, elle ne reconnaîtra qu'après ses «instructions au sosie » les capacités techniques qu'elle a développées presque en autodidacte, se focalisant au début uniquement sur les rapports ambivalents de son directeur. Cette focalisation, partagée par les autres secrétaires plus anciennes, constituera ainsi le symptôme principal des empêchements vécus dans ce travail. La compréhension de ce symptôme servira surtout comme exemple généralisable (Davydov, 1992) de la forme prise par les rapports entre salariés et responsables hiérarchiques, dont le service a été le théâtre. L'analyse montrera que les relations tendues - jusqu'à la maladie de Mme B., la secrétaire tombée en dépression - vécues par les salariés vis-à-vis de leurs hiérarchiques, étaient liées à une tentative de «mise en ordre» de la part de ces derniers de l'activité globale du service. Pendant des années, en effet, son fonctionnement était fondamentalement axé sur des relations de travail entre responsables du service et leurs subordonnés qui s'étaient révélées particulièrement pathogènes pour les salariés. 


\section{2.- Les discussions dans le collectif d'analyse pour en finir avec les passions tristes}

Les descriptions de l'activité subjective permises par les « instructions au sosie », discutées dans le groupe d'analyse, ont conduit à une nouvelle compréhension, collectivement menée, des relations subjectives des secrétaires les plus anciennes à leurs responsables. Reliant entre eux d'autres épisodes particuliers sur le thème que Mme C. avait fait émerger, l'analyse a montré une tendance très marquée dans ce service-là à un détournement du rapport salarial. Normalement ce rapport est censé instaurer une séparation entre ce qui appartient à l'entreprise et ce qui continue d'appartenir à la personne (Doray, 2011). Avant la réorganisation, les secrétaires les plus anciennes s'étaient trouvées mêlées à des relations de travail que leurs supérieurs hiérarchiques avaient trop chargées de dimension interpersonnelle. Ils instauraient ainsi avec l'un(e) ou l'autre secrétaire une dépendance affective cachée sous les formes d'une assistance spécifique à leur travail. Sans en avoir une conscience claire, les secrétaires acceptaient cette dépendance et les charges qu'elle comportait un peu parce qu'elles s'y sentaient contraintes, mais aussi pour se «distraire» de leur activité de secrétariat. Certaines tâches directement adressées aux usagers du service n'étaient pas réalisées ou trop retardées par manque de temps. Elles étaient parfois désinvesties car remplacées par des tâches jugées plus intéressantes, entre autres l'accompagnement du supérieur hiérarchique dans son activité dirigeante (congrès, colloques, réunions) au sein de l'organisme social.

Mais « l'autre pour soi », le dirigeant qui choisissait son ou sa secrétaire, pouvait disparaître de la scène. C'était le cas du responsable qui avait «élu » pour soi la secrétaire tombée malade, Mme B. L'analyse collective relèvera pour elle comme pour ses collègues un dysfonctionnement majeur dans les relations de travail. Pour Mme B., ce dysfonctionnement s'était traduit en une dépendance très gratifiante vis-à-vis de l'ancien directeur du service, parti à la retraite l'année où elle tombera, ensuite, malade. Il l'avait soutenue et protégée jusque-là, en cachant, par cette protection, les fragilités de ses savoir-faire, notamment en informatique, qu'elle n'avait pas pu dépasser. Face à la perception de ce manque et face à l'impossibilité d'un retour à un avant protecteur, la salariée avait d'abord choisi l'issue, inconsciente, de la maladie. Puis, la reconnaissance plus claire de cette dimension l'a poussée à choisir de quitter son emploi, départ pour lequel elle a négocié, à l'aide du syndicat, une indemnisation financière.

La recherche respective entre secrétaires et supérieurs hiérarchiques d'un autre "pour soi », censé tout pouvoir et tout vouloir (et tout donner) avait de fait secoué la dimension transpersonnelle du métier, mise à mal par une dérégulation du rapport collectif à l'activité commune. C'est en ce sens que trop «d'interpersonnel » a dévitalisé la dimension collective du métier, déréglant les critères d'efficacité propres au travail bien fait, toujours issus de ceux qui réalisent dans la durée le même métier. Lorsque cette dimension règle les collectifs de travail qui peuvent ainsi négocier ensemble leur place dans l'organisation globale, le métier peut se dire « en santé ».

\section{3.- Traces subjectives de l'élaboration des affects : le commentaire « officiel » de Mme C.}

Il nous semble possible, maintenant, de mieux comprendre la place que nous attribuons aux affects lorsqu'on intervient dans des situations où les conflits de travail semblent insolubles spontanément. D'un point de vue méthodologique l'analyse collective de l'activité subjective permet de transformer des épisodes très concrets en objets éveillant des affects (un exemple : la description de la chaise vide de Bruno). En s'arrêtant sur ces évènements, les émotions éveillées peuvent être dites, pour soi et pour les autres, et sont rendues ainsi compréhensibles et partageables. L'origine des sentiments ressentis est généralement clivée de la conscience à cause de censures que l'on s'impose. Pour ce motif le moyen narratif classique reste inadéquat. Par exemple, des entretiens individuels ou collectifs visant à rendre compte directement des causes des conflits n'offrent, le plus souvent, que des versions-écran aux sentiments éprouvés. 
Car les histoires que l'on (se) raconte pour argumenter les tensions ressenties voilent leur dimension réelle. On finit pour n'admettre qu'une version, se répétant du point de vue représentationnel en une seule traduction possible des affects éprouvés («si je ne suis pas invitée aux réunions, je me sens exclue $=$ je suis exclue ; si mon responsable ne vient plus discuter avec moi (la chaise vide de Bruno) $=$ je ne me sens plus reconnue $=$ je ne suis pas reconnue »). La dimension réelle de ces sentiments est bien plus complexe et, de plus, surdéterminée par des schèmes émotionnels qui se sont construits au cours de l'histoire personnelle et professionnelle. On peut accéder à ce réel non-pas par un accès direct verbalement exprimé - mais à travers des associations périphériques, souvent involontaires (Bucci, 1984, 1997 ; Merganthaler, 1996). C'est ensuite, au sein du collectif, que les affects émergés dans l'analyse de l'activité personnelle peuvent aboutir à une généralisation de leur signification, fournissant ainsi une nouvelle compréhension des conflits en acte.

Le commentaire de Mme C . à ses instructions au sosie peut être illustratif d'un premier mouvement de généralisation. Ce commentaire est adressé à l'ensemble des participants du dispositif d'analyse, les collègues de travail et les responsables hiérarchiques ${ }^{5}$. Son écriture est complètement habitée par cette adresse, qui en formate par conséquent le style et les choix de contenu. Les remarques succinctes que Mme C. produira dans son écrit ne montrent que les aspects dicibles publiquement de l'élaboration qu'elle a produite. Nous pensons, néanmoins, que cela constitue une trace valide et significative du processus d'élaboration qui lui a permis de reprendre la main sur sa situation professionnelle et de contribuer à une transformation de la situation collective.

Voici ce commentaire, qui contribuera à l'écriture d'un document plus général signé par l'ensemble du collectif des secrétaires, à la fin de l'intervention.

«Suite à mon entretien, quelques précisions et propositions.

Tout d'abord, une mise au point en ce qui concerne les «périodes froides » du journal. J'ai parlé du manque de travail, de la culpabilité qui s'y attache, mais je tiens à relativiser en reconnaissant que ces périodes sont, en fait, utilisées pour m'avancer sur d'autres choses : la prochaine maquette, la recherche pour la création de logos (dans le cadre du travail), l'auto formation sur les logiciels (photoshop, illustrator) dont je n'ai eu aucune formation. (...)

Je pense que pour mieux cerner le travail, la place que j'occupe, actuellement, au sein du journal et particulièrement dans le secteur «V.N.», il serait nécessaire, avant toute chose, que mes responsables hiérarchiques se situent eux-mêmes, à savoir Bruno, afin d'éviter le rôle «tampon » où je me trouve placée involontairement. Préciser la fonction de chacun, leurs responsabilités et le travail que je peux être amenée à réaliser à la demande de l'un ou de l'autre, sans risquer de froisser la susceptibilité de chacun, éliminerait quelques malentendus.

Pour revenir à «V.N. " et au travail particulier qui m'incombe, j'aimerais rappeler que si la dénomination de « secrétaire PAO » a, effectivement, mis en cohérence le travail accompli, cela ne m'a pas pour autant intégré dans l'équipe de fabrication du magazine. Il m'apparaît tout à fait négatif d'être cantonnée à un travail technique, d'où la vie même du magazine, semble être exclue. J'ai proposé à plusieurs reprises d'assister aux collectifs de rédaction de «V.N. » pour remédier à cette situation. Il semblerait que ce ne soit pas la place d'une « secrétaire PAO ».

La division du travail, je connais et je peux l'admettre quand les choses sont clairement définies mais là, on ne peut pas dire que cela soit limpide. Puisqu'il m'est permis, par l'intermédiaire de cette analyse du travail, de proposer des choses positives pour la réalisation de mon travail, j'insisterai donc pour renouveler ma demande de participation aux collectifs de rédaction de «V.N. », et particulièrement le dernier, celui qui précède le bouclage du magazine.

En fait, que la « secrétaire PAO » puisse connaître le déroulement de la fabrication du magazine ne

5 Sans pouvoir s'arrêter ici sur cette partie du travail d'analyse, ces derniers ont co-analysé leur activité de réorganisation du service. Cette analyse, réalisée avec les cliniciens de l'activité, a permis développer leur propre compréhension des conflits dont ils ont pu reconnaître les zones de responsabilité . 
peut, à mon sens, nuire à personne, et surtout ne remettre la place de personne en question, bien au contraire, cela ne pourrait que contribuer à rendre plus concret mon travail. J'ai l'impression de travailler « en marge » de la vie du magazine, de l'équipe. Les informations me parviennent au coup par coup, de manière aléatoire, ne serait-il pas plus simple de participer, d'avoir la communication en direct de l'équipe ? Cela ne remet pas en cause la retransmission de la secrétaire de rédaction, Marthe, qui est elle-même soumise à l'actualité du moment, mais le fait que je sois tributaire de la disponibilité de Marthe pour être informée, cela accentue le fait que je ne fais pas partie intégrante d'une équipe de travail. Et personne ne saurait retransmettre «la vie d'un magazine », soit on y participe, soit on exécute des tâches, là, oui, on est dans la division du travail !

Je ne demande pas à remettre en question le statut d'une «technique », je propose que l'on veuille bien réévaluer la méthode, l'organisation du travail, et que l'on prenne en considération la secrétaire PAO dans un processus de travail, et le sens que la fabrication du magazine a pour eux comme pour moi.

Ne plus être soumise à la décision arbitraire d'une personne qui décide si je dois savoir ou ne pas savoir. Je souhaiterais que cette décision soit prise collectivement, et que l'on puisse me communiquer officiellement si l'on juge utile que mon travail de secrétaire PAO se doit d'être limité, dans l'intérêt de la fabrication du magazine, à l'espace de la maquette sur ordinateur.

\section{8.- En guise de conclusion}

Il faut se demander : qu'est-ce qu'on élabore par l'usage de notre cadre méthodologique ? Une réponse possible est la suivante: l'élaboration concerne tout d'abord les expériences émotionnelles qui animent l'activité subjective, circulant dans les directions de l'activité qu'on avait définies plus haut. En effet, ce qu'on investit physiquement et psychiquement 1) dans la réalisation de la tâche prescrite, 2) dans les relations avec tous ceux qui ont affaire à cette tâche ; 3) ce que de cet investissement revient sur soi comme signe d'avoir pu ou pas travailler « comme il faut », ce sont des processus mobilisés par des affects, des émotions, des sentiments que tout individu au travail utilise pour agir et qui l'agitent. Le cadre d'analyse que nous utilisons organise la mise en débat de des re-présentations verbales (ou filmiques, si l'on utilise la méthode des auto-confrontations simples et croisées) de l'activité subjective dans le collectif de travail. Il stimule ainsi une activité dialogique mettant en confrontation les professionnels à la description de leur activité et à celle de leurs collègues. Les débats sur l'activité commune que ces méthodes provoquent dans le collectif de travail produisent, quand ce processus fonctionne, une transformation de la signification des sentiments vécus dans la situation. Cette transformation est, en partie, le résultat de l'élaboration subjective des émotions que l'analyse a mis au grand jour. C'était le cas, par exemple, de Mme C., qui a pu expliciter, à soi-même et aux collègues, l'ambivalence vécue vis-à-vis de sa dépendance à l'un des responsables du service. Si sa collègue, Mme B., lorsque son chef a pris sa retraite a fait une maladie de la perte de sa dépendance, Mme C. n'en a ressenti, dans un premier temps, que de l'amertume et de la haine. L'élaboration subjective s'étant déployée dans un cadre collectif, ces émotions ont pu migrer hors de la sphère personnelle. Relativisant la dimension interpersonnelle des conflits ressentis, Mme $\mathrm{C}$. et ses collègues ont pu les référer à la dimension qui soutient l'autoorganisation collective du métier, l'instance transpersonnelle. C'est quand cette dimension fait défaut que se défont les liens censés régler l'activité commune. Chacun doit alors se débrouiller comme il peut dans la situation, avec ses dilemmes, ses empêchements, ses peurs. Les salariés se « font organiser » sans intercaler entre les critères des gestionnaires et leur propre activité les critères retravaillés collectivement, forgés dans et par l'expérience directe du travail d'exécution. Suite à la co-analyse de leur activité, secondée par la conduite en parallèle d'une co-analyse de l'activité des différents responsables du service, les secrétaires ont pu re-dicter leurs propres critères de travail. Elles l'ont fait dans un document écrit collectivement et adressé à leur direction. Et le travail commun a retrouvé son souffle.

La signification d' «activité transférentielle » que nos méthodes de co-analyse sollicitent devrait 
à ce point être plus claire. Si l'activité d'analyse permet d'articuler les problèmes vécus personnellement à une dimension autre que celle des conflits interpersonnels, c'est grâce à la «traduction» des problématiques personnelles en problèmes de métier. L'activité transférentielle représente ces mouvements. Traduire, du latin trans-ducere, c'est étymologiquement transférer quelque chose d'une dimension à une autre. Ici ce sont les significations des sentiments personnels qui ont pu se transférer dans la dimension transpersonnelle, en réanimant ses formes organisationnelles.

\section{BIBLIOGRAPHIE}

Bucci, W. (1984). Linking words and things: Basic processes and individual variation. Cognition, 17, 137-153.

Bucci, W. (1997). Psychoanalysis and Cognitive Science. New York: Guilford Press [traduction italienne, Bucci W., Psicoanalisi e scienza cognitiva. Roma: Giovanni Fioriti editore, 1999].

Clot, Y. (1995). L'échange avec un sosie pour penser l'expérience : un essai. Société française, 53, 51-55.

Clot, Y. (1999). La fonction psychologique du travail. Paris: PUF.

Clot, Y. (2009). Travail et pouvoir d'agir. Paris: PUF.

Clot, Y. (2001). Méthodologie en clinique de l'activité. L'exemple du sosie. In M. Santiago Delefosse, \& G. Rouan (Eds.). Les méthodes qualitatives en psychologie (pp. 125-147). Paris: Dunod,

Cosnier, J. (1994). Psychologie des émotions et des sentiments. Paris: Retz.

Damasio, A (1999). Le sentiment même de soi : corps, émotions, conscience. Paris: Odile Jacob

Davydov, V. V. (1988). The concept of theoretical generalization and problems of educational psychology. Studies in East European Thought, 36(3), 169-202.

Doray B. (2011) Psychopathologie du travail : de la resymbolisation. Toulon: Erès.

Drida, M., Engel E., \& Litzenberger, M. (1998). Du harcèlement ou la violence discrète des relations de travail. In Actes $d u 2^{\circ} \mathrm{CIPPT}$, Paris.

Enriquez, E. (1997). Les jeux du pouvoir et du désir dans l'entreprise. Paris: Desclée de Brouwer

Gori, R. (2002). Logique des passions. Paris: Denoël.

Merganthaler, E. (1996). Emotion-abstraction patterns in verbatim protocols: a new way of describing psychoterapeutic process. Journal of Consulting and Clinical Psychology, 64, 1306-1315.

Oddone, I., Re, A., \& Briante G. (1981). Redécouvrir l'expérience ouvrière. Vers une autre psychologie du travail. Paris: éditions Sociales.

Pagès M. (1993). Psychothérapie et complexité. EPI, Desclée de Brower.

Rimé B. (2005). Le partage social de émotions. Paris: PUF.

Scheller, L. (2001). L'élaboration de l'expérience du travail. La méthode des instructions au sosie dans le cadre d'une formation universitaire. Clinique de l'activité et pouvoir d'agir, Éducation Permanente $146,161-174$.

Scheller, L. (2003). Élaborer l'expérience du travail : activité dialogique et référentielle dans la méthode des instructions au sosie. Thèse de doctorat, Paris, CNAM.

Scheller, L. (2004). Clinique de l'activité et élaboration de conflits dans le travail : une méthode d'action. In Paroles de praticiens et description de l'activité. Outils méthodologiques pour la formation (pp. 80-96). Bruxelles: De Boeck.

Vygotsky, L. (1998). Théorie des émotions. Étude historico-psychologique. Paris: l'Harmattan.

Vygotski, L. (2003). Conscience, inconscient, émotions. (Trad. F. Sève \& G. Fernandez). Paris: La Dispute.

\section{RESUME}

Certaines interventions en clinique de l'activité sont directement impulsées par une demande concernant des conflits apparemment insurmontables entre salariés et directions. Cet article rend compte de l'une de ces interventions, à partir d'un point d'entrée concernant le traitement des affects, des émotions et sentiments qui traversent et 
mobilisent l'activité de travail. Cette entrée constitue l'objet théorique de ce texte et l'objet clinique de l'intervention. L'article veut montrer comment à partir de l'activité d'analyse de la situation de travail, analyse qui mobilise des dialogues sur l'activité subjective et collective, prend forme une activité que nous avons définie de « transférentielle». Les résultats de l'intervention ont en effet conduit à transférer les affects personnels propres aux conflits destructeurs en cours en affects compris comme étant liés au délitement des formes transpersonnelles du métier analysé. Cela a permis une révision des formes d'organisation de l'activité du service, à partir de préconisations produites par le collectif des salariés, validés par le groupe des dirigeants.

MOTS CLEFS

activité de co-analyse, activité dialogique, activité transférentielle, affect, émotions, sentiments

\section{REFERENCEMENT}

Scheller, L. (2013). Travail, affects, activité transférentielle. Activités, 10(2), 249-260, http://www .activites.org/v10n2/v10n2.pdf

Article soumis le 2 janvier 2012, accepté pour publication le 28 avril 2013. 